\title{
Géomorphologie Dynamique: Analyse Systémique De La Morphodynamique Hydrique Continentale Par Ravinement
}

Dr. Seydou Alassane Sow,

Laboratoire Leïdi « Dynamiques des territoires et développement», Section de Géographie, Ecosystémes et Environnement, Université Gaston Berger de Saint-Louis, Sénégal

\section{Résumé}

L'histoire de la géomorphologie a connu une grande révolution paradigmatique avec les travaux de Wiliam Moris Davis. Il a défini le système géomorphologique comme l'ensemble de toutes les formes de la surface de la terre subissant des transformations et étant la fonction des trois variantes : structure, processus et temps. Cependant, le positionnement d'emblée des réflexions géomorphologiques dans l'analyse systémique conduit à élargir les problématiques du champ disciplinaire de cette science. Dans le cas de la géomorphologie dynamique, ce type d'analyse conduit à concevoir des recherches intégrées au sein des bassins versants afin de mieux mettre en lumière certains processus morphodynamiques. En l'absence de zone d'étude précise, l'étude est basée sur un balayage des connaissances bibliographiques à travers le domaine continental dans sa globalité. Ainsi, l'article montre que l'analyse systémique demeure une approche efficace pour lire en intégralité le fonctionnement des processus morpodynamiques hydriques continentales en géomorphologie, en particulier le ravinement. La caractérisation du ravinement sur un bassin versant grâce à l'analyse systémique doit se fonder sur trois caractéristiques essentielles: hydrologique, morphogénique et anthropique. Chaque critère correspondant à l'une des notions géographiques fondamentales : Temps, Espace et Société.

Mots clés : Géomorphologie, Analyse Systémique, Morphodynamique Hydrique, Ravinement, Bassin Versant 


\title{
Dynamic Geomorphology: Systemic Analysis of Continental Water Morphodynamics by Gullying
}

\section{Dr. Seydou Alassane Sow,}

Laboratoire Leïdi « Dynamiques des territoires et développement », Section de Géographie, Ecosystémes et Environnement, Université Gaston Berger de

Saint-Louis, Sénégal

\begin{abstract}
The history of geomorphology has undergone a great paradigmatic revolution with the work of Wiliam Moris Davis. He defined the geomorphological system as the set of all forms of the earth's surface undergoing transformation and being the function of three variants: structure, process and time. However, the immediate positioning of geomorphological reflections in systemic analysis leads to widening the problems of the disciplinary field of this science. In the case of dynamic geomorphology, this type of analysis leads to the design of integrated research within watersheds in order to better highlight certain morphodynamic processes. In the absence of a precise study area, the study is based on a sweeping of bibliographic knowledge across the continental domain as a whole. Thus, the article shows that systemic analysis remains an effective approach to fully read the functioning of continental water morphological processes in geomorphology, in particular gullying. The characterization of gullying on a watershed using systemic analysis must be based on three essential characteristics: hydrological, morphogenic and anthropogenic. Each criterion corresponding to one of the fundamental geographic concepts: Time, Space and Society.
\end{abstract}

Keywords: Geomorphology, Systemic Analysis, Water Morphodynamics, Gullying, Watershed

\section{Introduction}

L'historique de la géomorphologie dynamique expliquant l'évolution des formes de relief est structurée, selon Fort (2015), en trois grandes périodes : avant, pendant et après William Moris Davis (1850-1934) dont le concept du cycle d'érosion fut à son époque une grande révolution. En effet, selon les auteurs susmentionnés, les précurseurs (Hérodote, Aristote, et Sénèque,) estimaient que les reliefs terrestres s'inscrivaient dans le temps long, presque permanent et que les déformations de l'écorce terrestre n'étaient liées qu'aux 
séismes. Pour eux, contrairement aux variations saisonnières du climat, du couvert végétal ou du débit des rivières, les formes de relief semblaient immuables car leur évolution n'était guère perceptible à l'échelle d'une génération humaine.

Cette conception catastrophique de l'évolution des formes de relief fut réfutée aux XVIIIe et XIXe siècles par Hutton (1726-1797), Playfair (17481819) et Lyell (1797-1875) ${ }^{1}$ qui estiment que c'est l'action répétée de petits événements (reptation des sols, action des rivières, etc.) sur de très longues durées qui agit le plus sur l'élaboration des modelés, montrant ainsi le lien entre formes de relief et érosion : c'est le principe de l'actualisme qui stipule que le relief évolue depuis toujours sous l'action de forces érosives du même type que celles qui agissent actuellement. Selon ce principe, il y a uniformité des lois au cours du temps (exemple de la loi de gravité) et uniformité des processus d'évolution de relief. Ce principe sera complété par les idées de Surell (1841), Powell (1834-1902) et Gilbert (1843- 1918), qui précisent le rôle de la végétation et du substrat géologique dans l'évolution des formes de relief. Toutes ces théories serviront de base pour Davis (1850-1934) qui, à travers sa théorie sur le « cycle d'érosion » (1899), distingue trois stades : la jeunesse, la maturité et la vieillesse.

- Au stade de la jeunesse, les reliefs se caractérisent par des pentes fortes, sur lesquelles ruissellent torrents et rivières qui le creusent et l'accentuent au sens strict.

- Au stade de la maturité, les rivières continuent à inciser les reliefs tout en élargissant leurs vallées.

- Et le stade de la vieillesse est caractérisé par de faibles pentes créant une topographie très aplanie : la «pénéplaine », dominée par quelques reliefs résiduels ou glacis, à l'instar du plateau polygénique du Ferlo (ancienne vallée devenue fossile et située au milieu du Sahel sénégalais).

Cette théorie du cycle d'érosion stipule que ce sont les eaux, courantes et concentrées, qui contrôlent l'ensemble des processus d'érosion dans un contexte de région bioclimatique tempérée humide et exoréique, et stable tectoniquement.

Cependant, les détracteurs de Davis, mettent en évidence l'interaction constante entre tecto-orogenèse et morphogenèse, en montrant que certaines régions du globe connaissent un régime permanent de surrection, et que leur évolution est plutôt régie par un équilibre dynamique. Dans les décennies 1940 et 1950, on assiste à l'émergence de nouvelles théories en géomorphologie basées sur la quantification et la modélisation qui font recours à des lois 
physiques et à des modèles explicatifs (Peguy, 1942 ; Horton, 1945 ; Strahler, 1952, etc.). Aussi, la géomorphologie climatique parue avec Cholley (1950), Tricart et Cailleux (1965) qui définissent la notion de "système morphogénétique » en évoquant un complexe d'agents formant un «système » d'érosion propre à chaque climat.

Depuis la fin du XXe siècle, la géomorphologie prend en considération les activités humaines dans les processus d'évolution du milieu physique, pour devenir une science applicable et utile à la société : il s'agit des approches intégrales tenant à considérer les bassins versants comme des systèmes. La géomorphologie appliquée permet aujourd'hui de prendre en compte les changements sociétaux et d'avoir une approche intégrée entre les différents éléments du système morphogénétique et entre les divers compartiments des bassins versant et leurs relations longitudinales, horizontales, verticales et temporelles (Tricart, 1978).

Le bassin versant est l'unité de base sur laquelle travaille un géomorphologue continentalise. C'est à l'échelle de cet espace que se déroulent les processus de transferts de flux hydriques. A cet effet, des compilations de données de grande taille peuvent être produites afin de mettre en lumière les liens bassin versant - réseau hydrographique (ravins, torrents, chenaux, etc.) pour l'estimation des processus morphodynamiques comme le ravinement.

Selon Riou (1990), le ravinement est un processus de concentration des eaux sur un tracé linéaire et verticale. D'après un schéma classique, il apparait lors que s'approfondissent les entailles. Cette évolution est souvent due à une surexploitation du milieu. Les dimensions des ravins peuvent être impressionnantes : profondeur supérieur à $2 \mathrm{~m}$ ou à $10 \mathrm{~m}$, voire davantage ; largeur oscillant entre $10 \mathrm{~m}$ et $20 \mathrm{~m}$ et longueur entre $200 \mathrm{~m}$ et $1000 \mathrm{~m}$. Le ravin est ainsi un prolongement d'une entaille dans un matériel peu résistant et fournissant une alimentation par nappe de profondeur. Ceci est fréquent dans les grands manteaux d'altérites, à relief collinaire et accentué par la dégradation du couvert végétal.

Les ravins peuvent avoir des origines assez variées. Les seuls points communs étant la nécessaire concentration de l'eau, et une forte accumulation des pluies pendant une durée assez longue. Dans un bassin versant, à l'échelle de chaque ravin, le rythme d'évolution de la largeur varie suivant la section et la période où l'on se situe au niveau des axes hydrauliques. Il y a par conséquent une anisotropie de cette forme de morphodynamique hydrique : les propriétés du ravinement et des ravins varient donc suivant la direction des flux hydriques en action.

L'objectif de cet article est de montrer la place de l'analyse systémique dans la géomorphologie dynamique à partir d'une approche basée sur la morphodynamique hydrique par ravinement. En l'absence de zone d'étude 
précise, l'étude était basée sur un balayage des connaissances bibliographiques à travers le domaine continental dans sa globalité. Il procède à priori par une présentation théorique de l'analyse systémique, ensuite montre sa place dans la géomorphologie dynamique, et enfin établie une caractérisation de la morphodynamique par ravinement par l'analyse systémique.

\section{Théories sur l'analyse systémique : essai de définition}

Née aux Etats Unis au début des années 1950, connue et pratiquée en France depuis les années 70, l'analyse systémique ouvre une voie originale et prometteuse à la recherche et à l'action. La démarche a déjà donné lieu à de nombreuses applications, aussi bien en sciences physiques que sociales. Elle repose sur l'appréhension concrète d'un certain nombre de concepts tels que : système, interaction, rétroaction, régulation, organisation, finalité, vision globale, évolution, etc. Elle prend forme dans le processus de modélisation, lequel utilise largement le langage graphique et l'élaboration de modèles quantitatifs et qualitatifs (cartes, graphiques, modèles dynamiques, opérables sur ordinateur et débouchant sur la simulation).

C'est pourquoi la mise en œuvre de cette démarche passe par un effort d'apprentissage conceptuel et pratique auquel doivent consentir tous ceux (chercheurs, décideurs professionnels et politiques, hommes d'action mais aussi simples citoyens désireux de comprendre divers processus), qui ambitionnent de réaliser une plongée scientifique dans la complexité des processus, afin d'être capable dans un premier temps de s'y orienter, puis dans un second temps d'agir sur elle.

Combinant en permanence connaissance et action, l'analyse systémique se présente comme une conjugaison d'un savoir et d'une pratique. Selon l'Association Française des Sciences des Systèmes Cybernétiques, Cognitifs et Techniques (rappelée en 1994 par la Revue Internationale de Systémique), : l'analyse systémique regroupe les démarches théoriques, pratiques et méthodologiques, relatives à l'étude de ce qui est reconnu comme trop complexe pour pouvoir être abordé de façon réductionniste, et qui pose des problèmes de frontières, de relations internes et externes, de structure, de lois ou de propriétés émergentes caractérisant le système comme tel, ou des problèmes de mode d'observation, de représentation, de modélisation ou de simulation d'un phénomène ou processus complexe.

Pour appréhender la complexité des processus ou des phénomènes, l'analyse systémique fait appel à un certain nombre de concepts que l'on a appelé concepts fondamentaux de l'analyse systémique.

\subsection{Concepts fondamentaux de l'analyse systémique}

Quatre concepts fondamentaux structurent l'analyse systémique. 


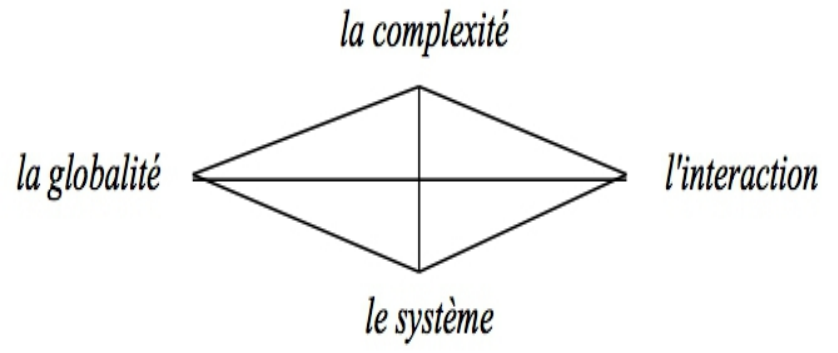

Figure 1 : Les dimensions de l'analyse systémique (Donnadieu et al., 2003)

\subsubsection{La complexité}

Comme il a été montré précédemment, la non prise de conscience de la complexité est la cause de la lente émergence de l'analyse systémique. Sans complexité, le rationalisme analytique pouvait sembler suffisant pour appréhender le monde et la science. Ce concept renvoie à toutes les difficultés de compréhension (flou, incertain, imprévisible, ambiguë, aléatoire) posées par l'appréhension d'une réalité complexe et qui se traduisent en fait pour l'observateur par un manque d'information (accessible ou non).

\subsubsection{Le système}

Ce concept constitue le socle sur lequel repose l'analyse systémique. Etymologiquement, le mot provient du grec sustêma qui signifie «ensemble cohérent ». Plusieurs définitions peuvent en être données et nous retiendrons deux définitions.

La définition «large » donnée par Jacques Lesourne : Un système est un ensemble d'éléments en interaction dynamique.

- La définition «étroite » donnée par Joël de Rosnay : Un système est un ensemble d'éléments en interaction dynamique, organisé en fonction d'un but. Cette définition met l'accent sur la finalité ou le but poursuivi par le système.

is les chercheurs :

- systèmes ouverts / systèmes fermés sur leur environnement,

- systèmes naturels / artificiels/ sociaux,

- systèmes organisés hiérarchiquement / systèmes en réseau.

\subsubsection{La globalité}

Il s'agit d'une propriété des systèmes complexes, souvent traduite par l'adage «le tout est plus que la somme des parties» et selon laquelle on ne peut les connaître vraiment sans les considérer dans leur ensemble. Cette globalité exprime à la fois l'interdépendance des éléments du système et la cohérence de l'ensemble. Mais ce concept pourtant riche est malheureusement 
souvent traduit superficiellement par la formule vague «tout est dans tout». Sous le nom d'approche globale, le concept désigne également la voie d'entrée dans l'analyse systémique. On entend par là qu'il convient d'aborder tous les aspects d'un problème progressivement, mais non séquentiellement : partir d'une vue générale (globale) pour approfondir les détails, avec de nombreuses itérations et retours en arrière pour compléter ou corriger la vision antérieure.

\subsubsection{L'interaction}

Ce concept complète celui de globalité. Il s'intéresse à la complexité au niveau élémentaire de chaque relation entre les constituants du système pris deux à deux. Initialement emprunté à la mécanique où l'interaction se réduit alors à un jeu de forces, la relation entre constituants se traduit le plus souvent dans les systèmes complexes, par un rapport d'influence ou d'échange portant aussi bien sur des flux de matière et d'énergie.

La notion d'interaction déborde largement la simple relation de cause à effet qui domine la science classique. Et connaître la nature et la forme de l'interaction est plus importante pour le systémicien que de connaître la nature de chaque composant du système.

\section{L'analyse systémique : une méthode}

L'analyse systémique est non seulement un savoir, mais aussi une pratique, une manière d'entrer dans la complexité. La pédagogie à mettre en œuvre doit être novatrice tant dans sa démarche générale que dans les outils employés.

\subsection{La démarche générale de l'analyse systémique}

La démarche se déroule par étapes : observation du système par divers observateurs et sous divers aspects ; analyse des interactions et des chaînes de régulation; modélisation en tenant compte des enseignements issus de l'évolution du système ; simulation et confrontation à la réalité (expérimentation) pour obtenir un consensus. Une telle démarche doit être à la fois prudente et ambitieuse :

- prudente en ce qu'elle ne part pas d'idées préétablies mais de faits qu'elle constate et que l'on doit prendre en compte;

- ambitieuse en ce qu'elle recherche la meilleure appréhension possible

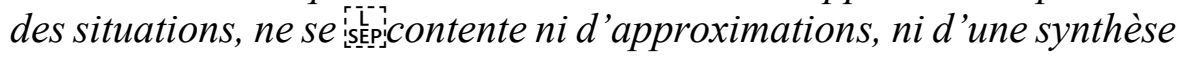
rapide, mais vise à comprendre et à enrichir la connaissance.

\subsection{Les outils de l'analyse systémique}

Nous présenterons dans cette section trois outils de base, avant de faire une ébauche sur le langage graphique, qui est la langue naturelle de l'analyse systémique, puis d'exposer la modélisation qui, mieux qu'un outil, est au cœur 
même de l'approche systémique.

\subsubsection{La triangulation systémique}

Remarquablement adaptée à la phase d'investigation d'un système complexe, la triangulation va observer celui-ci sous trois aspects différents mais complémentaires, chacun lié à un point de vue particulier de l'observateur.

L'aspect fonctionnel est surtout sensible à la finalité ou aux finalités du système. On cherche spontanément à répondre aux questions : que fait le système dans son environnement ? A quoi sert-il ?

L'aspect structural vise à décrire la structure du système, l'agencement de ses divers composants. On retrouve là la démarche analytique avec cependant une nuance de poids : l'accent est mis bien davantage sur les relations entre composants que sur les composants eux-mêmes, sur la structure que sur l'élément.

L'aspect historique (ou génétique ou dynamique) est lié à la nature évolutive du système, doté d'une mémoire et d'un projet, capable d'auto-organisation. Seule, l'histoire du système permettra bien souvent de rendre compte de certains des aspects de son fonctionnement. Pour les systèmes sociaux, c'est même par elle qu'il convient de démarrer l'observation.

Naturellement, la triangulation systémique se développe en combinant ces trois voies d'accès. Plus exactement, on se déplace d'un aspect à un autre au cours d'un processus en hélice qui permet, à chaque passage, de gagner en approfondissement et en compréhension, mais sans que jamais on puisse croire que l'on a épuisé cette compréhension.

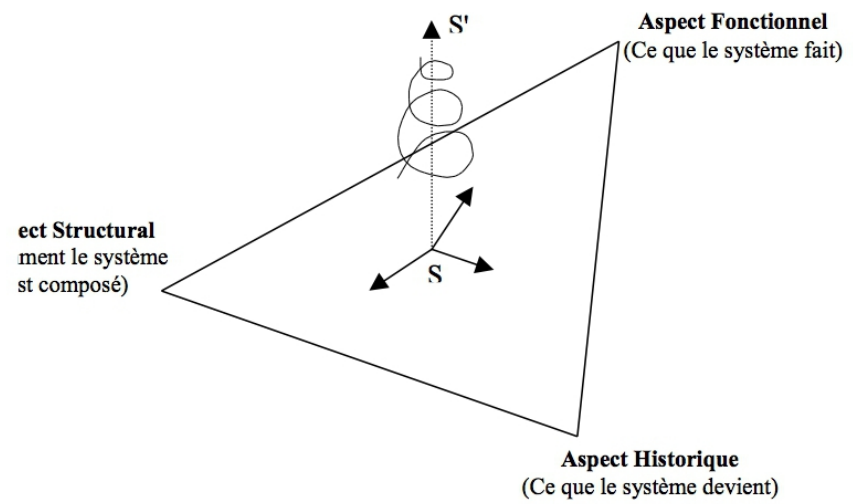

Figure 1 : Les différents aspects de la triangulation systémique 


\subsubsection{Le découpage systémique}

A la différence de la décomposition analytique, on ne cherche pas à descendre au niveau des composants élémentaires mais à identifier les soussystèmes (modules, organes, sous-ensembles, etc.) qui jouent un rôle dans le fonctionnement du système. Cela suppose de définir clairement les frontières de ces sous-systèmes (ou modules) pour faire ensuite apparaître les relations qu'ils entretiennent entre eux ainsi que leur finalité par rapport à l'ensemble. On remarquera que ce problème de la frontière se pose aussi pour le système lui- même : comment le définir par rapport à son environnement, quel découpage?

La question du découpage s'accompagne toujours d'un certain arbitraire et ne peut recevoir de réponse univoque. Cependant, pour réaliser le découpage de la manière la plus pertinente possible, on peut s'appuyer sur quelques critères, suggérés d'ailleurs par la systémique elle-même, les deux premiers repris de la triangulation:

- le critère de finalité : quelle est la fonction du module par rapport à l'ensemble?

- le critère historique : les composants du module partagent-ils une histoire propre? ?[isp?

- le critère du niveau organisation : par rapport à la hiérarchie des niveaux šẹpid'organisation, où se situe le module étudié ?

- le critère de la structure : certaines structures ont un caractère répétitif et se retrouvent à plusieurs niveaux d'organisation. On parle dans ce cas de structures fractales ou en hologrammes. Pour analyser ces structures, il suffit alors de s'intéresser à un seul de ces hologrammes que l'on va soumettre à un grossissement connu sous le nom de zoom ou effet de loupe.

Cet effet de loupe est d'une large utilisation. Il importe néanmoins de rester conscient de ses limites. La démarche postule en effet l'existence, dans le système de redondances ou régularités reliées au tout par une relation de circularité. Et il n'est pas sûr que ces conditions soient toujours et partout réalisées.

\subsubsection{Le langage graphique}

Le langage graphique est largement utilisé dans le domaine technique (la carte universellement employée, et qui est la représentation commode d'un territoire, fait partie de ce langage graphique). Notons qu'il s'agit bien d'un véritable langage, à côté des langages naturels discursifs, écrits ou parlés, et $\mathrm{du}$ langage mathématique formel. Tous ces langages recourent d'ailleurs volontiers au langage graphique par des schémas et idéogrammes ainsi que par la géométrie et la théorie des graphes. is sêpi On attribue quatre avantages au 
langage graphique :

- il permet une appréhension globale et rapide du système représenté (après apprentissage),

- il contient une forte densité d'informations dans un espace limité (économie de moyens),

- il est monosémique et semi-formel (faible variabilité d'interprétation),

- - il possède une bonne capacité heuristique (notamment dans un travail de groupe). [s.

\subsubsection{La modélisation}

Modéliser est d'abord un processus technique qui permet de représenter, dans un but de connaissance et d'action, un objet ou une situation voire un événement réputé complexe. On l'utilise dans tous les domaines scientifiques concernés par la complexité des processus ou des phénomènes étudiés. isepí:Mais la modélisation est aussi un art par lequel le modélisateur exprime sa vision de la réalité. En ce sens, on peut parler de démarche constructiviste. La même réalité, perçue par deux modélisateurs différents, ne débouchera pas nécessairement sur le même modèle. Toutefois, si le modélisateur souhaite que son modèle soit opératoire, c'est-à-dire permettre à l'utilisateur de s'orienter dans la complexité et d'agir efficacement sur elle, il doit prendre en compte certains critères et respecter certaines lois de construction. istep:

Cet outil peut ainsi être utile pour l'application de l'analyse systémique dans les sciences de la géographie physique, en particulier la géomorphologie dynamique.

\section{L'analyse systémique dans la géomorphologie dynamique}

L'analyse systémique dans la géomorphologie est apparue à travers les travaux de Wiliam Moris Davis (1850-1934) qui a défini le système géomorphologique comme : l'ensemble de toutes les formes de la surface de la terre subissant des transformations, et étant la fonction des trois variantes : structure,processus et temps. Par la suite, ce système a été précisé et modifié. La notion de structure comprend un ensemble de relations entre des éléments particuliers, celle du temps, les changements irréversibles et de longue durée, la fonction, enfin, n'est rien d'autre que la réaction des formes du relief face aux changements intérieurs et extérieurs.

L'étude des processus géomorphologiques à l'aide de l'analyse systémique permet de faire ressortir un nombre important de facteurs, de rapports complexes existant entre eux, une influence réciproque, ainsi que la rétroaction. Cette approche pourrait servir à l'établissement d'un schéma du développement des processus ou, à plus large échelle, à la détermination des 
rapports entre les facteurs qui en conjugaison participent au façonnement des formes.

Pour ce qui est du cas spécifique de la géomorphologie dynamique en domaine continentale, le but à atteindre est la prévision ou l'estimation en terme de bilan d'un phénomène donné. Cette prévision peut être soit générale, apportant des informations sur les réactions d'un système donné à des changements du milieu (environnement), soit détaillée, déterminant les étapes de la morphogenèse, apportant une caractéristique quantitative, et faisant ressortir les relations fonctionnelles et, à partir de là, indiquant la direction de changements basée sur une interaction de facteurs particuliers. L'effet final d'une interaction n'est pas la somme d'actions partielles, et le processus unique, entièrement responsable du développement du relief n'existe pas dans la nature. L'ensemble (façonnement des formes) représente beaucoup plus qu'une somme de facteurs (processus).

L'érosion hydrique (un de ces processus) consiste en une destruction du matériel lithologique par un mouvement progressif de courants de flux hydrique. Au moment où commence ce processus, plusieurs facteurs agissent. Tout essai d'explication de la façon dont se fait ou se fera le processus d'érosion, et de l'intensité avec laquelle ce processus transformera une zone continentale nécessiterait une présentation systémique de facteurs dont dépend ledit processus (érosion hydrique).

Le degré de simplification de ces facteurs peut être présenté sur l'exemple suivant : si l'on voulait faire figurer les microcycles d'érosion accompagnant la morphodynamique hydrique de fond de vallées dans un modèle mathématique, il faudrait remplacer le processus par les caractéristiques suivantes : géométrie du courant hydrique attaquant une berge (amplitude et hauteur), énergie avec laquelle le courant attaque (détruit) la berge exposée. Pareillement, tous les autres facteurs devraient être déterminés de façon plus formalisée. Il en résulterait, alors, que le degré d'influence sur le développement de la morphodynamique hydrique est différent pour chacun d'eux.

A l'échelle de la région sahélienne du Sénégal (Nord), les observations de terrain concernant le développement du processus d'érosion hydrique sur les piedmonts des glacis confirment pleinement cette observation. La même physiographie du milieu (géométrie et pente), les même conditions climatiques et hydrologiques (cinématique du courant hydrique) n'empêchent pas l'apparition de différences quant à la vitesse du développement de la morphodynamique hydrique façonnant le relief. Ces différences sont dues, à leur tour, aux différences de structures géologiques, de couvertures végétales, et de la façon d'aménager le terrain.

Ainsi, la question qu'un géomorphologue pourrait se demander est la suivante : comment serait-il possible de pronostiquer à l'aide de l'analyse 
systémique sur le développement du processus d'érosion hydrique dont le ravinement en particulier?

\section{Morphodynamique hydrique par ravinement et analyse systémique}

Cette section essaye de faire une caractérisation du ravinement par l'analyse systémique. Elle sera cependant précédée d'une esquisse en guise de bilan scientifique sur le processus géomorphologique (ravinement).

\subsection{Esquisse d'un bilan scientifique sur le processus géomorphologique (ravinement)}

Le ravinement prend son origine quand le ruissellement s'organise et provoque une érosion concentrée. L'évolution surfacique de l'érosion hydrique concentrée peut suivre un schéma classique, classifiés d'après leur hiérarchie en amont (séquence griffes ou griffures, rigoles, ravineau et ravines torrentielles (Lilin et Koohafkan, 1987)) ou en aval (rivière et fleuve).

Les éléments qui contrôlent l'érosion par ravinement sont : le volume et la vitesse du ruissellement, la résistance du sol au cisaillement et la friabilité du régolithe (Roose, 1991). Le volume ruisselé est fonction de la surface du bassin, du degré de saturation des sols et de leur capacité d'infiltration, de l'intensité et volume des pluies, de la dynamique hydrogéologique du bassin. Tandis que la vitesse du ruissellement est fonction de la pente, de la rugosité des surfaces et des aménagements (infrastructures ou végétation).

L'érosion par ravinement est l'un des mécanismes de dégradation des paysages le plus intense et difficile à contrôler. L'incision des roches par ruissellement concentré représente une source de sédiments importante, et elle peut attendre jusqu'à $90 \%$ de l'érosion totale d'un bassin versant (Mathys et Poesen, 2005).

La quantité de sédiments produits dans les bassins versants soumis à une forte érosion par ravinement en amont peut causer de graves et coûteux problèmes en aval, dans les différentes œuvres d'ingénierie comme les canaux et les barrages. Ce type de dégradation du paysage peut causer aussi des événements violents comme les glissements ou les mouvements de terrain, ce qui met en risque de catastrophes naturelles la population des zones montagneuses (Pereira, 2009).

Hauge (1977) et Poesen (1993) séparent les rigoles et les ravines saisonnières par une approche purement bidimensionnelle : une section transversale critique de $929 \mathrm{~cm}^{2}$ (le critère du pied carré, square foot). D'autres critères de séparation incluent une largeur et une profondeur minimum d'environ 0,3 m et de 0,6 m (Brice, 1966) ; ou bien celle de Imeson et Kwaad (1980) : une profondeur minimum de $0,5 \mathrm{~m}$. Par rapport à la limite supérieure des ravines, aucune définition précise n'existe, la frontière entre une grosse ravine et une rivière reste très vague (Poesen et al., 2003). 
Riou (1990) propose la différenciation sémantique et morphologique suivante :

- Rigole : Profondeur $=$ de quelques centimètres à 40 ou à $50 \mathrm{~cm} /$ Largeur $=$ de quelques décimètres à $2 \mathrm{~m} /$ Longueur $=$ échelles métriques et décamétriques.

- Ravine : Profondeur $=0,5$ à $1,5 \mathrm{~m}$ voire $2 \mathrm{misEp} / /$ Largeur $=5$ à $10 \mathrm{~m}$, avec un mini-talweg de 1 à $2 \mathrm{~m}$ environ / Longueur $=20$ à $100 \mathrm{~m}$ ou plus.

- Ravin : Profondeur $=$ supérieure à $2 \mathrm{~m}$ et jusqu'à $10 \mathrm{~m}$ ou plus $/$ Largeur $=10-20 \mathrm{~m} /$ Longueur $=200-1000 \mathrm{~m}$

Brochot et Meurnier (1995), mentionnent que les ravines sont des thalwegs permanents en forme de $\mathrm{V}$ symétrique, où les processus d'érosion sont dus au ruissellement concentré et aussi aux éboulements, à la solifluxion pelliculaire, au ruissellement diffus et aux mouvements de masse.

La transition entre les différentes échelles de manifestation superficielle d'érosion hydrique concentrée représente un continuum : des griffes ou griffures, des rigoles, des ravineaux, des ravines saisonnières, des ravines torrentielles, des rivières. N'importe quel système de classification des formes d'érosion hydrauliques concentrée est d'une certaine façon subjective (Grissinger, 1996).

La plupart des processus géomorphologiques ont une origine gravitationnelle, par conséquent le taux et la vitesse de l'eau ou tout autre mouvement de masse est une fonction de la pente plus la texture de la matière qui se déplace. Les détections de changements spatiaux-temporels de la pente permettent de décrire de futurs déplacements de matière. Ces changements fournissent une information valable sur le rapport entre le flux d'entrée et de sortie de matière (Etzelmüller, 2000).

$\mathrm{Au}$ regard de ces informations, une représentation 3D général d'un bassin versant permettra une meilleure connaissance de la dynamique spatiale et temporelle des différents facteurs qui contrôlent la dégradation des paysages par ravinement. Aussi bien comme outil d'aide à la compréhension du processus érosif (à une échelle grossière), que comme outil d'aide à la prise de décision dans un programme de gestion des risques associés.

L'analyse numérique des dérivés du relief dans un Système d'Information Géographique (SIG) permettra, à partir d'un certain nombre de seuils critiques de chaque facteur de contrôle, de diviser le paysage d'un bassin versant en fonction de différents types d'érosion (Bishop et al., 2003), de cartographier la géomorphologie pour l'interprétation structurale, de quantifier des changements superficiels, ou de faire une analyse régionale de paysages (Bolongaro-Crevenna et al., 2004). 
Les indicateurs d'érosion superficielle les plus utilisés pour cartographier l'étendue de l'érosion du sol sont les rigoles et les ravines. Bien que ces manifestations ne puissent pas être individuellement identifiées par les capteurs satellitaire ou aérien (à échelle grossière) jusqu'à ce qu'elles atteignent de grandes dimensions, il est possible d'obtenir l'information de façon indirecte à travers les facteurs environnementaux qui contrôlent leur développement sur le terrain (King et al., 1989).

Les formes linéaires de manifestation de l'érosion concentrée possèdent un lien entre les échelles spatiale et temporelle d'autant quelles correspondent à une évolution naturelle du processus d'érosion par ravinement sur le terrain. Par exemple, si on veut observer des érosions fines comme les griffures, la fréquence d'acquisition des données doit être forte.

Le volume des travaux par les hydrologues et les forestiers aux échelles spatio-temporelles plus grossière est significativement plus important dans la littérature que pour les échelles plus détaillées (Daba et al., 2003). Ces travaux sont souvent dirigés vers l'étude des systèmes de ravines torrentielles et leurs dynamiques de transport des sédiments. Néanmoins, il y a aussi un fort intérêt dans l'étude de ravinement associé aux catastrophes naturelles, par des géologues et géomorphologues (Casson et al., 2005 ; Demoulin, 2006 ; Nichol et al., 2006).

Bien que les processus de base soient fondamentalement différents, le mouvement de masse et le ravinement du paysage partagent certaines caractéristiques communes (Zinck et al., 2001). Le sol conditionne fréquemment le déclenchement et le développement de l'érosion et, en même temps, est affecté par eux. Les deux processus ont de multiples origines, les mécanismes de déclenchement pourraient avoir lieu à la surface de terrain ou en dessous. Ces phénomènes chaotiques, déclenchés par le changement soudain de l'équilibre environnemental et ils peuvent produire des dommages catastrophiques.

La matrice de contrôle de la morphodynamique hydrique par ravinement est le résultat de l'interaction d'une série de facteurs de site et de facteurs « externes ». Les facteurs externes sont les conditions météorologiques générales et locales, ou micro-climat (Descroix et Mathys, 2003). Les facteurs de site sont la topographie, le type de sol ou de matériel géologique dégradé (régolithe), et la couverture de la terre, ajoutons aussi les œuvres anthropiques. En effet, diverses sortes d'activités humaines, pratiques agricoles, exploitations forestières, pâturages, construction des routes et bâtiments tendent à modifier les phénomènes d'érosion, en l'accélérant souvent de façon considérable: d'où l'importance de l'analyse systémique dans l'étude des processus géomorphologiques. 


\subsection{Caractérisation du ravinement par l'analyse systémique}

Le positionnement des réflexions géomorphologiques dans l'analyse systémique conduit de plus en plus à élargir les problématiques du champ disciplinaire de la géomorphologie. Dans le cas des processus morphodynamiques dont le ravinement, ce type d'analyse conduit à concevoir des recherches intégrées au sein de bassins versants (Schumm, 1977): territoire intégrateur de différents processus physico-humains évoluant dans le Temps et l'Espace, sous l'effet des influences des Sociétés (Sow, 2018).

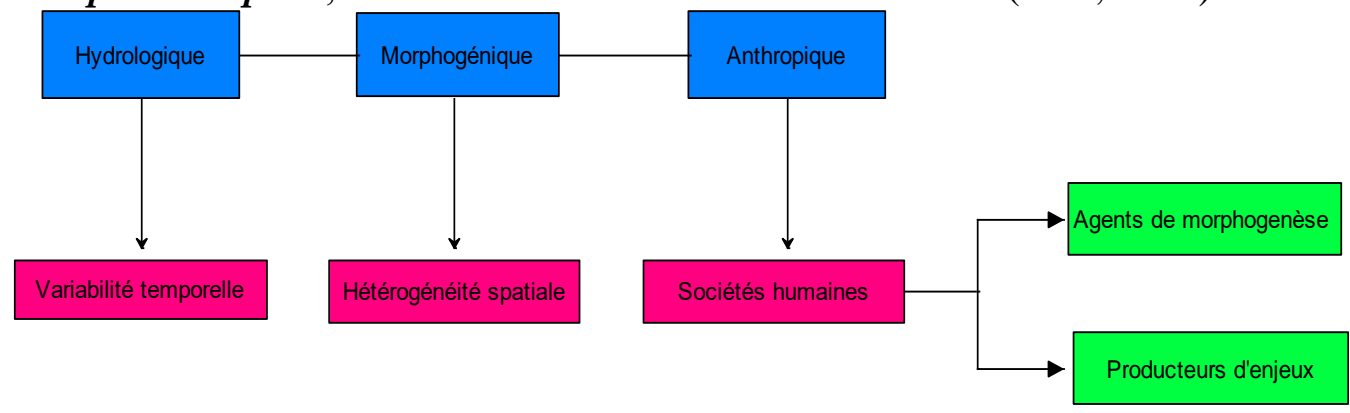

Lire : en bleu = critéres de définition; en rose = caractéristiques des critéres et en vert $=$ propriétés des sociétés humaines

Figure 2 : Modèle de caractérisation de l'espace géographique : application sur un bassin versant

Cette figure 2 propose un modèle de caractérisation de l'espace géographique (bassin versant), en se basant sur trois caractéristiques essentielles: hydrologique, morphogénique et anthropique. Chaque critère correspond à l'une des notions géographiques fondamentales : Temps, Espace et Société.

- L'hétérogénéité spatiale fait appel à la notion d'espace. L'espace est le lieu dans lequel se déroulent les différents processus. Il est défini par des coordonnées géographiques. Il a des caractéristiques propres qui évoluent sous l'action des agents en jeux.

- La variabilité temporelle évoque l'idée de temps. Ce dernier est considéré dans cette approche comme l'unité de base, pour l'analyse des processus physiques et anthropiques à l'origine du ravinement dans l'espace géographique. Le temps est défini selon les lois géomorphologiques et hydrologiques en ère, épisode, séquence, ou événement.

- Les sociétés humaines constituent les Hommes et leurs œuvres. Ces derniers agissent de façon double. Ils influencent le processus (ravinement) : ils sont des agents morphogéniques. Ils créent des enjeux sur l'espace géographique : ils subissent les conséquences du 
processus, qui se manifestent ici en termes d'inondations et de perte en terre.

La caractérisation du ravinement par l'analyse systémique fait appel à quelques notions géographiques fondamentales que sont : Espace, Temps et Société, qu'il faudra mettre en relation avec la dynamique du processus (ravinement).

\subsubsection{Ravinement et Espace}

L'évolution de la morphodynamique hydrique par ravinement se réalise toujours selon des emboitements spatiaux, qu'il convient de ne pas perdre de vue pour comprendre les conséquences qui en découlent (Sow, 2019).

A cet effet, un classement des échelles géographiques allant des éléments englobant de grande taille vers les éléments de taille plus réduite, inscrits dans les précédents devient une nécessité. Un plateau peut se décomposer en éléments tabulaires et en vallées ; les éléments tabulaires en collines et en dépressions; les collines en versants variés; les versants en espaces plans, bourrelets et ruptures de pentes et sur ces espaces plans peuvent exister des ravines. Un versant est lui-même un emboitement de formes : une ravine s'inscrit dans un espace plan ou concave n'évolue pas à la même vitesse (Ciattoni et Veyret, 2003).

D'ores et déjà, le bassin versant, forme élémentaire et se traduisant par une topographie plus ou moins plane, qui joint un interfluve à une plaine alluviale ou à un talweg, apparait comme espace géographique privilégié pour mettre en évidence le ravinement. Se positionnant entre le sixième et le septième ordre de grandeur il peut être l'unité de base sur laquelle travaille un géomorphologue ou un hydrologue. C'est à l'échelle de cette espace que se déroulent les processus de transferts (Valadas, 2004) des flux sous l'effet de la morphodynamique hydrique dont le ravinement.

La démarche systémique propose alors de caractériser les éléments physiographiques et les processus hydrologiques, de les mesurer et les expliquer pour comprendre leurs dynamiques d'évolution, en s'intéressant aux mécanismes qui les créent et aux vitesses auxquelles elles évoluent. Au-delà de l'indispensable connaissance de chacun des mécanismes responsables pris isolément, il faut appréhender la façon dont ces derniers se combinent entre eux, et dont ils interagissent avec les processus anthropiques au niveau de l'espace géographique : d'ou l'originalité de l'approche.

L'analyse systémique demeure particulièrement importante pour l'étude du ravinement dans un espace comme le bassin versant. En effet, pour Annie REFFAY (1996), la géomorphologie dynamique se veut être une géomorphologie quantitative, au sens plein du terme, attachée, non seulement 
à la mensuration des formes, mais aussi à l'estimation de la vitesse d'élaboration des processus à l'échelle de l'espace géographique. Le bassin versant demeure en ce sens le résultat d'un bilan et de relations complexes entre des phénomènes de morphogénèse et d'anthropogenèse (physiques et anthropiques).

La morphodynamique hydrique par ravinement s'inscrit toujours dans un contexte temporel.

\subsubsection{Ravinement et Temps}

Tenir compte des échelles temporelles en géomorphologie est une nécessité dans l'analyse systémique. Les héritages géomorphologiques sont à prendre en compte pour comprendre les dynamiques actuelles (Valadas, 2004). C'est souvent le caractère éphémère de leur déroulement qui caractérise le plus les morphodynamiques hydriques actives dont la torrentialité ou le ravinement. Une lave de ravinement s'observe en quelques minutes, une montée de crue en quelques heures, etc. Le temps long en géomorphologie, c'est surtout celui de la géomorphologie structurale qui crée, à l'échelle des millions d'années, les grandes formes en creux et en saillie qui forment les unités majeures du relief. En géomorphologie dynamique, la temporalité est relativement réduite, surtout quand les dynamiques sont vives. A cet effet, se digitalisent parfois dans l'espace géographique des moments d'excessivité ou de crise, qui traduisent un danger pour les populations : c'est l'aléa représenté par une inondation par exemple. Les aléas constituent des épisodes «normaux » de la dynamique terrestre y compris lorsque leurs caractères violents peuvent sembler anormaux ou extraordinaires pour les populations (Ciattoni et Veyret, 2003).

A certains moments, l'homme a perçu la nature en termes de régularité, de permanence, ce qui lui a permis d'effectuer certains choix d'aménagements. Dardel rappelle en 1952 que «l'homme, n'a pu s'affranchir des contraintes de la faim, de la soif et du froid, de la distance et de l'exubérance végétale, que parce qu'il pouvait compter sur la constance des phénomènes et sur l'invariance des phénomènes périodiques ». Or, la biophysique terrestre n'offre pas la régularité à laquelle l'établissement des moyennes climatiques, hydrologiques,... pourrait laisser d'ampleur et de fréquence très variables. Plus que la répétition du phénomène, ce sont les temps de retour qui sont importants : plus ceux-ci sont longs et plus la mémoire humaine a effacé le souvenir de l'épisode précédent et la surprise et l'imprévoyance aggravent les effets.

L'appropriation de l'analyse systémique permettra de recadrer le phénomène ravinement dans le contexte actuel de la dégradation des conditions bioclimatiques. Cette dégradation est marquée par une disparition progressive de la couverture végétale sous l'effet de la sévérité du contexte 
bioclimatique et d'une urbanisation progressive des bassins versants jadis ruraux en particulier. Ce processus est à l'origine des changements d'usages des sols observés et de la hausse des vitesses d'écoulement des eaux à l'échelle des bassins versants (Mahé et Paturel, 2013 et Decroix et al., 2013).

\subsubsection{Ravinement et Société}

Pour exister, toute société humaine a besoin d'un espace qui lui préexiste et qu'elle transforme. Sa survie dépend de ses capacités à s'approprier celui-ci, à y survivre, à agir sur lui isolément ou avec d'autres. Toutes les sociétés produisent un ou des espaces organisés que l'on désigne sous le nom d'espace géographique.

L'espace géographique est à la fois produit des pratiques sociales et composante de ces pratiques. En mobilisant des démarches aussi fondamentales que : l'appropriation, l'exploitation, la communication et l'habitation, l'œuvre humaine de spatialisation ou de mise en espace de l'étendue terrestre est par conséquent partie intégrante de tout projet social. Elle présente pour chacune des sociétés une composante incontournable de son fonctionnement (Ciattoni et Veyret, 2003).

L'intégration du facteur social pour une caractérisation du ravinement par l'analyse systémique demeure particulièrement importante, car l'espace géographique est aussi social. Selon Veyret (1998), l'Homme, par ses diverses actions et notamment en modifiant les couverts végétaux peut contribuer à aggraver ou à déclencher certains processus d'érosion. Il agit indirectement sur le ruissellement le long des pentes, le ravinement à travers les axes hydrauliques, l'écoulement dans les talwegs, et sur les mouvements de masse (particules solides en charriage). Il contribue à accroitre des risques, qui dans certains milieux font l'objet d'études et de zonage, rendant nécessaire une connaissance précise des dynamiques, de la fréquence des aléas et de leur intensité.

Si aujourd'hui, l'Homme se donne l'illusion d'échapper aux contraintes naturelles, il demeure tout de même obligé de prendre en compte un nombre important de paramètres, en particulier ceux qui régissent l'évolution des modelés dans lesquels il vit. L'urbanisation rapide se soucie peu des conditions dans lesquelles elle se développe. Toutefois, même s'il est de moins en moins soumis et s'il sait se protéger des dynamiques naturelles, il doit encore souvent composer avec la nature, ne serait-ce qu'en termes d'aléas et de risques (Valadas, 2004).

La démarche du géomorphologue repose sur l'explication de l'évolution des unités, articulée avec les principaux changements au cours des temps, l'état actuel et enfin les processus de façonnement futurs en rapport avec le domaine morphoclimatique et les différentes formes d'anthropisation Sy (2013). 
L'analyse systémique met ainsi en exergue les différents usages que les sociétés font sur les formes et les formations superficielles. Elle traduit les types de pressions que les populations exercent sur la nature, et identifie ces dernières en tant que facteurs qui influencent et qui subissent les conséquences du phénomène ravinement.

\section{Conclusion}

L'appréhension de la morphodynamique hydrique par ravinement à travers les champs disciplinaires de la géomorphologie n'est pas une question facile à aborder. La variabilité spatiale du phénomène, le grand nombre de facteurs intervenant directement ou indirectement dans son explication, la difficulté, enfin, de passer d'une échelle d'analyse à une autre constituent autant de paramètres, qui complexifient le processus à l'échelle des bassins versants. L'analyse systémique demeure à cet effet une approche efficace pour lire en intégralité le fonctionnement des processus morphodynamiques continentaux en particulier le ravinement. Cette approche (de part l'introduction de nouveaux outils, de nouveaux concepts, de nouveaux moyens informatiques permettant d'opérer des sauts en avant, de tester les idées existantes, et donc d'ouvrir la voie vers la formulation de nouvelles hypothèses explicatives) a abouti à une sorte de rupture épistémologique en géomorphologie dynamique, notamment à travers l'introduction de nouveaux paradigmes qu'il convient davantage d'explorer.

\section{References:}

1. Aliti, B. (2020). Géomorphologie et dynamique des paysages du bassin versant du Zio (Sud-Togo). Thèse de Doctorat, Université de Lomé, Togo, 260p.

2. Bishop, M., Shroder, J., \& Colby J. (2003). Remote sensing and geomorphometry for studying relief production in high mountains. Geomorphology, (55), 345-361.

3. Bolongaro-Crevenna, A., Torres-Rodríguez, V., Sorani, V., Frame, D. \& Ortiz, M.A. (2004). Geomorphometric analysis for characterizing landforms in Morelos State, Mexico. Geomorphology, (7), 407-422.

4. Brice, J.B., (1966). Erosion and deposition in the loess-mantled Great Plains, Medecine Creek drainage basin, Nebraska. U.S. Geological Survey Professional Paper 352H, 235- 339.

5. Brochot, S. \& Meurnier M. (1995). Erosion de Badlands dans les Alpes du sud, BVRE de Draix, Cemagref Editions, 141-174.

6. Casson, B., Delacourt, C., \& Allemand, P. (2005). Contribution of multi-temporal remote sensing images to characterize landslide slip surface - Application to the La Clapière landslide (France). Natural Hazards and Earth Sciences, (5) 425-437. 
7. Ciattoni A. et Veyret Y. (2003). Les fondamentaux de la géographie. Armand Colin / VUEF, 219 p.

8. Daba, S. (2003). Assessment of gully erosion in eastern Ethiopia using photogrammetric techniques. Catena, (50), 273-291.

9. Davis, W. M., (1899). The geographical cycle. Geographical Journal, (14), 481-504.

10. Demoulin, J. (2006). Monitoring and mapping landslide displacements: a combined DGPS-stereophotogrammetric approach for detailed short- and long-term rate estimates. Terra Nova, (4), 290298.

11. Descroix, L. (2013). La crue de 2012 à Niamey : un paroxysme du paradoxe du Sahel ?. Sécheresse, (24), 3-13.

12. Etzelmüller, B. (2000). On the quantification of surface changes using grid-based digital elevation models (DEM). Transactions in GIS, (2), 129-143.

13. Fort, M. (2015). Géomorphologie dynamique et environnement. Armand Colin, Collection U, 335 p.

14. Grissinger, E. (1996). Rill and gullies erosion. In : Agassi, M. (Ed.), Soil Erosion, Conservation, and Rehabilitation. Marcel Dekker, New York, 153- 167.

15. Hauge, C., (1977). Soil erosion definitions. California Geology, (30), $202-203$.

16. Imeson, A.C, et Kwaad, F.J. (1980). Gully types and gully prediction. KNAG Geografisch Tijdschrift, (5), 430-441.

17. King, C., Maucorps, J., Aumonier, R., Renaux, B., \& Lenotre, N. (1989). Détection d'indices d'érosion par SPOT dans les sols limoneux du Nord-Pas de Calais, une étude multi-date. Bull. SFPT, 114, 10-13.

18. Lilin, C., et Koohafkan, A. P., (1987). Techniques biologiques de conservation des sols en Haïti, FAO, 36 p.

19. Mathys, N., et Poesen, J., (2005). Ravinement en montagne : processus, mesures, modélisation, régionalisation. Géomorphologie : relief, processus, environnement, 11 (1), 3-6. http://geomorphologie.revues.org/index187.html.

20. Mahe G. et Paturel E. (2009). 1896-2006 Sahelian annual rainfall variability and runoff increase of Sahelian Rivers. Geoscience, Volume 341, 538-546.

21. Nichol, J.E., Shaker, A., \& Wong, M.S. (2006). Application of highresolution stereo images to detailed landslide hazard assessment. Geomorphology, (76), 68-75.

22. Pereira J. A. (2009). MNT à très haute résolution spatiale pour la représentation $3 \mathrm{~d}$ de ravines d'érosion en montagne. Thèse de doctorat, 
Paris Tech, Institut des Sciences et Industries du Vivant et de l'Environnement (AgroParisTech), $260 \mathrm{p}$.

23. Poesen, J. (1993). Gully typology and gully control measures in the European loess belt. In : Wicherek, S. (Ed.), Farm Land Erosion in Temperate Plains Environment and Hills. Elsevier, Amsterdam, 221239.

24. Poesen, J., Nachtergaele, J., Verstraeten \& Valentin, C. (2003). Gully erosion and environmental change : importance and research needs. Catena, (50) 91-133.

25. Riou, G., (1990). L'eau et les sols dans les géosystèmes tropicaux, Masson, Paris 221p. SEEP?

26. Roose, E., (1991). Conservation des sols en zones méditerranéennes. Synthèses et proposition d'une nouvelle stratégie de lutte antiérosive : la GCES. Cah. Orstom, sér. Pédol., (2), 145-181.

27. Schumm, S. A., (1956). Evolution of drainage systems and slopes in badlands at Perth Amboy. Geol. Soc. America Bull., (67), 597-646.

28. Schumm S. A. (1977). The Fluvial System, Wiley, New York, 338p.

29. Sow S. A. (2018). Morphodynamique hydrique par ravinement et ses conséquences dans le bassin versant de Ourossogui, nord du Sénégal. Thèse de doctorat, Université Gaston Berger de Saint-Louis du Sénégal, 321p.

30. Sow S. A. (2019). Caractérisations granulométrique et paléoenvironnementale des sédiments d'un bassin versant à forte dynamique structurale dans un contexte de ravinement : bassin versant de Ourossogui (Nord du Sénégal). European Scientific Journal, Vol.15, No.32, 86-104.

31. SY A. A. (2013). Dynamiques sédimentaires et risques actuels dans l'axe Saint-Louis-Gandiol, littoral Nord du Sénégal. Thèse de doctorat de $3^{\text {ème }}$ cycle, Université Gaston Berger de Saint-Louis du Sénégal, $328 \mathrm{p}$.

32. Tricart, J., (1978). Géomorphologie applicable, ed. Masson, Paris, 204p.

33. Valadas B., (2004). Géomorphologie dynamique, Armand Colin, Paris, $192 \mathrm{p}$.

34. Veyret Y. (2001). Géo-environnement, Paris, Armand Colin, 159 p.

35. Zinck, J.A., López, J., Metternicht, G.I., Shrestha, D.P. \& VázquezSelem L. (2001). Mapping and modelling mass movements and gullies in mountainous areas using remote sensing and GIS techniques. JAG. 3(1), 43-53. 